\title{
Гибридные режимы работы термоэлектрических модулей
}

\author{
( И.А. Драбкин, Л.Б. Ершова \\ $\mathrm{OOO}$ „PMT“, \\ 115230 Москва, Россия \\ E-mail: igordrabk@gmail.com
}

Поступила в Редакцию 18 августа 2021 г.

В окончательной редакции 25 августа 2021 г.

Принята к публикации 25 августа 2021 г.

Предлагается не ограничиваться экстремальными режимами работы при конструировании термоэлектрических охладителей. В некоторых случаях удобным оказывается использование гибридного режима, который состоит из комбинации экстремального режима максимального холодильного коэффициента для больших разностей температур на модуле и общего режима охлаждения для малых.

Ключевые слова: термоэлектрические модули, гибридный режим, холодопроизводительность ветви.

DOI: 10.21883/FTP.2022.01.51804.06

\section{1. Введение}

Обычно в стационарной работе термоэлектрического модуля используются два режима: режим максимальной энергетической эффективности (режим максимального холодильного коэффициента $\left.\varepsilon_{\max }\right)$ и режим максимальной холодопроизводительности $Q_{0 \max }[1,2]$, различающиеся прежде всего величиной тока через охладитель. Режим работы модуля определяет конструкцию теплообменника на его горячей стороне, поэтому переход из одного режима работы в другой не ограничивается изменением тока через модуль, а требует внесения конструктивных изменений в этот теплообменник. Такая связь режимов работы модуля с конструкцией охладителя говорит в пользу того, что ограничиваться двумя режимами работы модуля не всегда бывает целесообразно. Особенно это заметно в режиме $\varepsilon_{\max }$ по мере приближения разности температур на модуле $(\Delta T)$ к 0. При этом, хотя холодильный коэффициент $\varepsilon$ растет обратно пропорционально $\Delta T$, холодопроизводительность $Q_{0 \varepsilon}$ стремится к 0 пропорционально $\Delta T$. В режиме $Q_{0 \max }$ при $\Delta T \rightarrow 0$ величина $\varepsilon \rightarrow 0.5$, а $Q_{0 \max }$ стремится к величине, которая определяется как максимальная холодопроизводительность $Q_{\max }$ модуля в этом режиме. В данной работе рассмотрены режимы работы термоэлектрического модуля, в дальнейшем называемые гибридными и не относящиеся к вышеперечисленным экстремальным режимам, а также показаны возможные области их применения.

\section{2. Исследование гибридных режимов}

Основная задача в области охлаждения заключается в обеспечении заданной величины $Q_{0}$ при так же заданной величине $\Delta T$ [3]. В экстремальных режимах ток через модуль определяется геометрией ветви и ее термоэлектрическими характеристиками, а нужную величину $Q_{0}$ обеспечивает количество пар в модуле. На рис. 1 кривые $Q_{0}$ дают представление о холодопроизводительности термоэлектрической ветви в экстремальных режимах в единицах $Q_{\max }$.

Из рис. 1 видно, что по мере уменьшения разности температур на модуле расхождение между величинами $Q_{0}$ в режимах $\varepsilon_{\max }$ и $Q_{0 \max }$ растет. При этом отношение $Q_{0 \varepsilon \max } / Q_{0 \max }$ по мере уменьшения $\Delta T$ стремится к нулю. Ясно, что для обеспечения необходимой холодопроизводительности модуля при $\Delta T \rightarrow 0$ в режиме $\varepsilon_{\max }$ должно расти до бесконечности количество ветвей в модуле. В этом плане режим $Q_{0 \text { max }}$ существенно лучше, однако потребляемая мощность и выделяемое тепло будут существенно выше, чем в режиме $\varepsilon_{\max }$.

Рассмотрим гибридные режимы работы ветви, обеспечивающие величину $Q_{0}$, лежащую в промежутке между холодопроизводительностями в режиме $\varepsilon_{\max }$ и $Q_{0 \text { max }}$. Так как условия экстремальности отсутствуют, их можно заменить любыми другими - например, задать желаемые зависимости

$$
\varepsilon=f(\Delta T),
$$

т. е. режим заданного холодильного коэффициента. Можно задаться также желаемой холодопроизводитель-

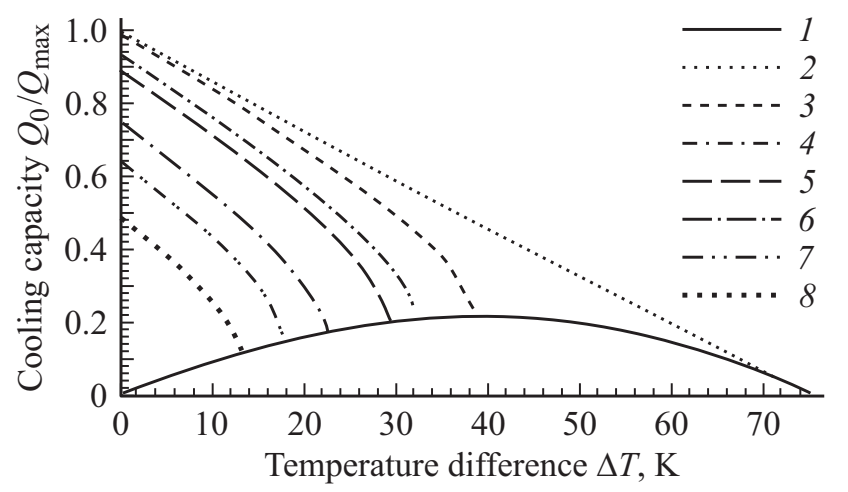

Рис. 1. Отношения холодопроизводительностей $Q_{0} / Q_{\max }$ при $Z=0.003 \mathrm{~K}^{-1}$ для экстремальных и гибридных с различными значениями $\varepsilon$ режимов работы: 1 - режим $\varepsilon_{\max } ; 2-$ режим $Q_{0 \max } ; \varepsilon=0.6(3), 0.85(4), 1(5), 1.5(6), 2(7), 3(8)$. 
ностью:

$$
Q_{0}=Q_{\max } \phi(\Delta T) .
$$

В (1) и (2) $f(\Delta T)$ и $\phi(\Delta T)$ - некоторые произвольные безразмерные функции и $Q_{\max }$ - максимальная холодопроизводительность ветви, равная

$$
Q_{\max }=\frac{\alpha^{2} T^{2}}{2 R} .
$$

Разумеется, эти функции должны удовлетворять условиям

$$
\begin{gathered}
\varepsilon_{\max }(\Delta T) \geq f(\Delta T) \geq \varepsilon_{Q_{0} \max }(\Delta T), \\
Q_{0 \max }(\Delta T) \geq Q_{\max } \phi(\Delta T) \geq Q_{\varepsilon \max }(\Delta T),
\end{gathered}
$$

где $\varepsilon_{Q_{0} \max }(\Delta T)$ - холодильный коэффициент в режиме $Q_{0 \max }$, а $Q_{\varepsilon \max }(\Delta T)$ - холодопроизводительность в режиме $\varepsilon_{\max }$.

Рассмотрим поведение $Q_{0}$ и $\varepsilon$ при задании условия (1). Уравнение теплового баланса на холодном конце ветви с температурой $T_{0}$ есть

$$
\alpha I T_{0}-\frac{1}{2} I^{2} R-K \Delta T=Q_{0},
$$

где $\alpha-$ термоэдс материала ветви, $I-$ ток через ветвь, $R$ - сопротивление ветви, $K$ - теплопроводность ветви, $Q_{0}$ - холодопроизводительность ветви.

Воспользовавшись определением холодильного коэффициента $Q_{0}=\varepsilon(I R+\alpha \Delta T) I[1]$, заменив в (6) соответствующую величину и решая уравнение (6) относительно $I$ при заданных значениях $\Delta T, f(\Delta T)$ и температуре горячего конца ветви $T$, получаем

$$
I=I_{q \max } F,
$$

где $I_{q \max }$ - ток в режиме $Q_{0 \max }$ при $\Delta T=0$, равный

$$
I_{q \max }=\frac{\alpha T}{R},
$$

а фактор $F$ равен

$$
\begin{aligned}
& F=\frac{1-(\Delta T / T)(1+f(\Delta T))}{1+2 f(\Delta T)} \\
& \times\left[1+\sqrt{1-\frac{\Delta T}{T} \frac{2(1+2 f(\Delta T))}{Z T(1-(\Delta T / T)(1+f(\Delta T)))^{2}}}\right],
\end{aligned}
$$

где $Z-$ термоэлектрическая эффективность $Z=\frac{\alpha^{2}}{R K}$.

Холодопроизводительность $Q_{0}$ записывается как

$$
Q_{0}=2 Q_{\max } \varepsilon\left[F^{2}+(\Delta T / T) F\right] .
$$

Корень в выражении (9) обращается в 0 при значениях $f(\Delta T)$, соответствующих максимальному холодильному коэффициенту, потому что значения $f(\Delta T)$, превышающие $\varepsilon_{\max }$ при заданном $\Delta T / T$, не имеют смысла в соответствии с (4).

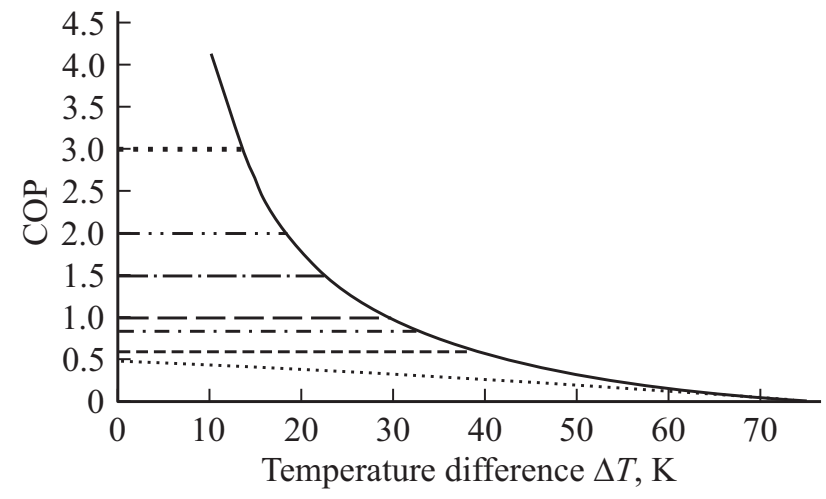

Рис. 2. Зависимости холодильного коэффициента $\varepsilon$ для $Z=0.003 \mathrm{~K}^{-1}$ от разности температур для экстремальных и гибридных режимов с фиксированным значением $\varepsilon$. При разностях температур, лежащих справа от пересечения прямых, соответствующих фиксированному значению $\varepsilon$ с кривой для режима $\varepsilon_{\max }$ используется режим $\varepsilon_{\max }$, а для температур слева от точки пересечения - режим фиксированного $\varepsilon$. Обозначения те же, что и на рис. 1. COP - coefficient of performance.

Выражения (9) и (10) справедливы не только для ветви, но и для пары, если под $I_{q \max }, Q_{\max }$ и $Z T$ понимать соответствующие величины для ветви или пары.

Рассмотрим в качестве примера случай $f=$ const. Coответствующие расчеты по формуле (10) для различных значений $\varepsilon$ представлены на рис. 1.

Из рисунка видно, что расчетные кривые для гибридных режимов отходят от кривой $\varepsilon_{\max }(\Delta T)$. Второй реперной точкой для кривых при $\Delta T=0$ и соответствующем значении $F$ является холодопроизводительность $Q_{00}$, значение которой можно получить из (10):

$$
Q_{00}=Q_{\max } \frac{8 \varepsilon}{(2 \varepsilon+1)^{2}} .
$$

Из рис. 1 видно также, что расчетные кривые перекрывают весь диапазон значений $Q_{0}$, расположенный между $Q_{0 \varepsilon} \max$ и $Q_{0 \max }$. Таким образом, при разностях температур, бо́льших $\Delta T_{\varepsilon \max }$, где $\Delta T_{\varepsilon \max }$ соответствует разности температур, при которых достигается максимум на кривой холодопроизводительности в режиме максимального холодильного коэффициента, холодопроизводительность совпадает с $Q_{0 \varepsilon} \max$, а при $\Delta T<\Delta T_{\varepsilon} \max$ выражение для холодопроизводительности дается общим выражением (10). На рис. 2 приведены соответствующие зависимости холодильных коэффициентов для экстремальных и гибридных режимов работы. Видно, что использование гибридных режимов позволяет заметно увеличить холодопроизводительность для небольших разностей температур, сохраняя при этом $\varepsilon$ на приемлемом уровне.

Рассмотрим работу ветви в режиме заданной холодопроизводительности (2), именно такой подход позволяет решить проблему с недостаточной холодопроизводительностью в режиме $\varepsilon_{\max }$ и обеспечить работу ветви 


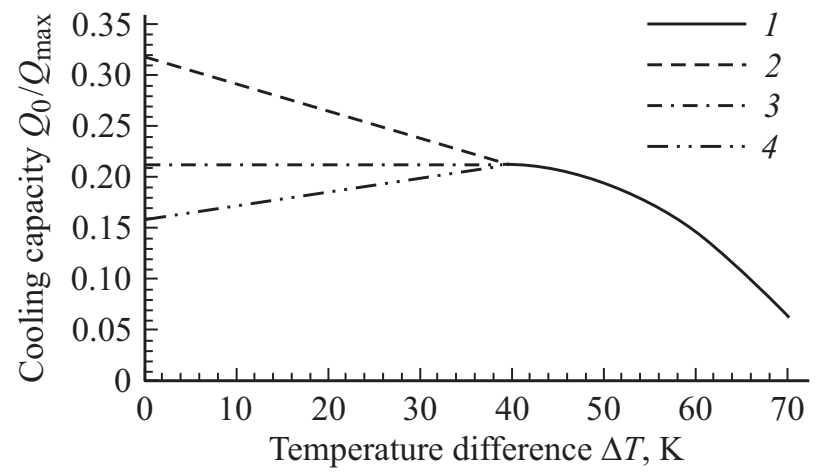

Рис. 3. Холодопроизводительность модуля в единицах $Q_{0} / Q_{\max }$ в условиях работы для температур $\Delta T>\Delta T_{\varepsilon \max }-$ режим максимального холодильного коэффициента, а для температур $\Delta T<\Delta T_{\varepsilon \max }-$ в режиме линейно меняющейся холодопроизводительности при $Z=0.003 \mathrm{~K}^{-1}$. Режимы работы: $1-$ режим $\varepsilon_{\max }, 2-Q_{0}=1.5 Q_{0 m}, 3-Q_{0}=Q_{0 m}$, $4-Q_{0}=0.85 Q_{0 m}$.

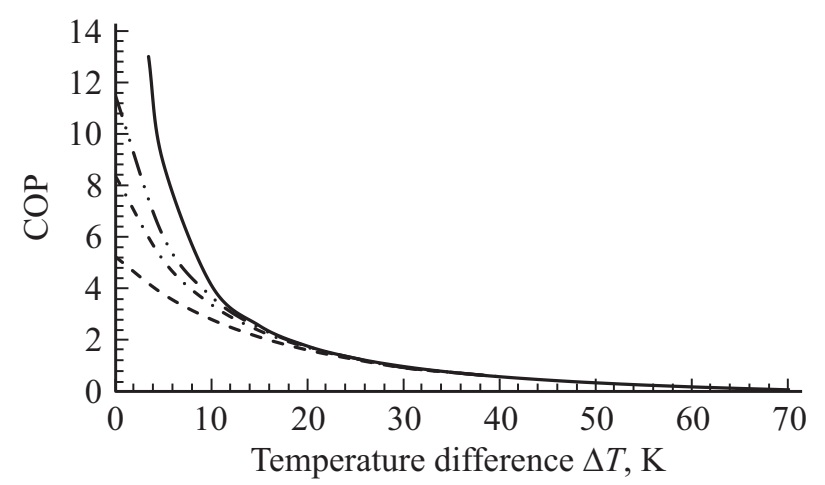

Рис. 4. Холодильный коэффициент для работы в различных условиях холодопроизводительности. Обозначения те же, что на рис. 3 .

c $\varepsilon<\varepsilon_{\max }$, но с заметно большей холодопроизводительностью. В этом случае фактор $F$ вместо (9) приобретает вид $F_{1}$ :

$$
F_{1}=\frac{T_{0}}{T}\left(1-\sqrt{1-\frac{2}{Z T_{0}^{2}}\left(\Delta T+\frac{Z}{2} T^{2} \phi(\Delta T)\right)}\right) .
$$

Рассмотрим простейший случай, когда $\phi$ изменяется c $\Delta T$ по линейному закону. Пусть максимум холодопроизводительности в режиме максимального холодильного коэффициента равен $Q_{0 m}$ и расположен при разности температур на модуле $\Delta T_{m}$, что соответствует температуре холодного конца $T_{0 m}$. При эффективности термоэлектрического материала $Z=0.003 \mathrm{~K}^{-1}$ величина $\Delta T_{m}=39.61 \mathrm{~K}$ и $Q_{0 m}=0.212159 Q_{\max }$. Выражение для $\phi$ приобретает вид

$$
\phi=\frac{Q_{0 m}}{Q_{\max }}\left(1+k\left(1-\frac{\Delta T}{\Delta T_{m}}\right)\right),
$$

где $k-$ некоторый коэффициент пропорциональности. Из (13) следует, что при $\Delta T=0 Q_{00}=Q(1+k)_{0 m}$, что дает возможность легко вычислить по (11) холодильный коэффициент при $\Delta T=0$. При $k=0$ величина $Q_{0 m}$ не зависит от температуры и равна в нашем случае $0.212159 Q_{\max }$.

Для случая (13) выражение для $F_{1}$ с учетом $[1,3]$ $Q_{0 m}=Q_{\max } \frac{\Delta T_{m}}{T^{2}} \frac{2\left(M_{m} T_{0 m}-T\right) M_{m}}{\left(M_{m}+1\right)\left(M_{m}-1\right)^{2}}$ приобретает вид

$F_{1}=$

$=\frac{T_{0}}{T}\left(1-\sqrt{1-\frac{2}{Z T_{0}^{2}}\left(\Delta T+\frac{\left(1+k\left(1-\Delta T / \Delta T_{m}\right)\right) Z \Delta T_{m}\left(M_{m} T_{0 m}-T\right) M_{m}}{\left(M_{m}+1\right)\left(M_{m}-1\right)^{2}}\right)}\right)$,

где $M_{m}=\sqrt{1+Z\left(T_{m}+\frac{\Delta T_{m}}{2}\right)}$, и холодильный коэффициент для $\Delta T<\Delta T_{m}$

$$
\varepsilon=\frac{\Delta T_{m}\left(M_{m} T_{0 m}-T\right) M_{m}\left(1+k\left(1-\Delta T / \Delta T_{m}\right)\right)}{T^{2}\left(F_{1}^{2}+\frac{\Delta T}{T} F_{1}\right)\left(M_{m}+1\right)\left(M_{m}-1\right)^{2}},
$$

а ток через модуль дается выражением $I=I_{q \max } F_{1}$.

Результаты расчетов приведены на рис. 3 и 4.

Из рис. 3 и 4 видно, что режим заданной холодопроизводительности дополняет режим заданного холодильного коэффициента и обеспечивает более широкие возможности для управления режимами охлаждения в области $\Delta T<\Delta T_{m}$.

\section{3. Заключение}

Предложенный гибридный режим дает возможность управлять холодопроизводительностью ветви и не привязывать эту величину к значениям при экстремальных режимах работы. Это полезно при проектировании охладителей, предназначенных для работы при разностях температур на модуле, которые меньше температуры, при которой достигается максимум холодопроизводительности в режиме $\varepsilon_{\max }$, и особенно в режиме работы при малых разностях температур. Кроме того, переход в гибридный режим оставляет произвол в выборе величины $\varepsilon$. Этим можно воспользоваться и попытаться оптимизировать конструкцию по каким-то другим параметрам, например по весу, габаритам или по стоимости.

\section{Конфликт интересов}

Авторы заявляют, что у них нет конфликта интересов.

\section{Список литературы}

[1] Э.М. Лукишкер, А.Л. Вайнер, М.Н. Сомкин, В.Ю. Водолагин. Термоэлектрические охладители (М., Радио и связь, $1983)$ c. 27.

[2] A.A. Melnikov, V.G. Kostishin, V.V. Alenkov. J. Electron. Mater., 46 (5), 2737 (2017).

[3] R. Marlow, E. Burke. In: CRC Handbook of Thermoelectrics, ed. by D.M. Rowe (London, CRC Press, 1995) p. 597.

Редактор Л.В. Шаронова 


\section{Hybrid operating modes of thermoelectric modules}

\section{I.A. Drabkin, L.B. Ershova}

RMT Ltd.,

115230 Moscow, Russia

Abstract It is suggested that thermoelectric coolers designing should not be limited to the extreme modes of their operation. In some cases, it is convenient to use so called hybrid modes a combination of the extreme mode of maximum coefficient of performance for large temperature differences and a general cooling mode for small ones. The proposed hybrid mode makes it possible to control the cooling capacity of the module and not to confine this value to that under the extreme operating conditions, the maximum coefficient of performance in particular. 